\title{
Preliminary study on a kinetic energy recovery system for sailing yachts
}

\author{
Giuseppe Leo Guizzi ${ }^{\mathrm{a}}$, Michele Manno ${ }^{\mathrm{a}, *}$, Guido Manzi ${ }^{\mathrm{b}}$, Marco Salvatori ${ }^{\mathrm{a}}$, \\ Domenico Serpellab \\ ${ }^{a}$ Dept. of Industrial Engineering, University of Rome Tor Vergata, Italy \\ ${ }^{b}$ Labor srl, Tecnopolo Tiburtino, Via G. Peroni 386, 00131 Rome, Italy
}

\begin{abstract}
This paper presents the preliminary theoretical results obtained on a model of a kinetic energy recovery system for sailing yachts, based on the conversion of wave-induced boat oscillations (heave, pitch and roll) into electric energy by means of a linear generator.

The recovery system is based on a linear generator, with a mass oscillating along the vertical axis and gaining kinetic energy: the resulting mechanical energy can be extracted (by means of electromagnetic damping) and converted into electricity. The oscillating mass incorporates permanent magnets which, moving in proximity of stator windings, generate electric power due to electromagnetic induction.

The device aims at recovering as much kinetic energy as possible from the natural movements of a sailing yacht on the sea, therefore taking the view of a boat as a moving wave energy converter with energy harvesting capability. The boat's motions can be vertical oscillations due to the buoyancy in the presence of sea waves, both when the boat is still or sailing, and rolling and pitching motions originated both by sailing in wavy waters and by the normal boat dynamics due to the sails' propulsion. Linear generators will convert kinetic energy into electrical energy to be used as "green" electricity for any possible application on board.
\end{abstract}

\footnotetext{
${ }^{*}$ Corresponding author

Email addresses: guizzi@ing.uniroma2.it (Giuseppe Leo Guizzi), michele.manno@uniroma2.it (Michele Manno), g.manzi@labor-roma.it (Guido Manzi), marco.salvatori87@gmail.com (Marco Salvatori), d.serpella@labor-roma.it (Domenico Serpella)
} 
Preliminary calculations show that a properly configured system could be able to recover approximately $100 \mathrm{~W}$ under most sea conditions on an almost continuous basis, which can be an extremely attractive result since an electric energy availability of $1-2 \mathrm{kWh}$ on a sailing yacht is of significant interest.

Keywords: Wave Energy Recovery, Linear Generator, Sail Yacht, Kinetic Energy Recovery System, SEAKERS

\section{Introduction}

This paper presents the preliminary theoretical results obtained on a model of a kinetic energy recovery system to be used on board of sail yachts in order to recover energy from the wave-induced boat's vertical motion (SEAKERS: SEA Kinetic Energy Recovery System).

Such a system would be able to recover actual free energy, as opposed to other devices, already commercially available, that subtracts energy from the propulsion offered by the wind's lift on the sails, as in the case of micro-wind turbines installed on the boat, which are set into motion by the apparent wind originating from the yacht's motion.

This system could address a well known unsatisfied requirement of yacht owners, since energy is a resource of primary importance in a boat, especially in a sailing one: it is well known that during a one-day cruise, electricity consumption has to be carefully managed (for instance the refrigerator is switched off), so as not to be short of energy at night. It often happens that, after one day of sail cruise, it is necessary to recharge the batteries through the on-board generator, which means keeping it on for hours, producing very annoying noise, smoke and pollution.

In practical terms, the SEAKERS device is intended to be a linear oscillator, with a mass oscillating along the vertical axis and gaining kinetic energy; if the mass is the moving element of a linear generator, the resulting mechanical energy can be extracted and converted into electricity. The oscillating mass incorporates permanent magnets which, moving in proximity of stator windings, generate electric power due to electromagnetic induction.

Basically, two emerging fields represent the conceptual background for this work: wave energy conversion, with particular reference to single-body heaving buoys with linear wave energy converters [1-6], and energy harvesting from ambient vibration $[7,8]$. 
In particular, the idea of using a linear generator originates from work carried out at the University of Uppsala [9-13], where such devices have been designed and tested in order to recover wave energy from a buoy, oscillating on the sea surface, connected to a rope that makes a piston move inside a generator placed on the sea floor. In the SEAKERS project, the oscillating mass is set into motion not directly by the sea waves but by its inertia as the yacht is subject to heave, pitch and roll motions: in this respect the system acts like an energy harvester, even though on a bigger scale if compared to the generators usually taken into consideration in this field, which usually provide electric power of tens or thousandths of microwatts [14].

In order to design and prototype the generator, it is necessary to set up a reliable model of different sea conditions that could be of practical interest for a normal cruise on a sail yacht (thus there is no need to consider extreme, stormy waves) and of the ship motion due to such sea states. Furthermore, it is important to find out, by means of a linearised model of the generator, how much power could be extracted for different operating conditions, in order to decide whether the project's outcome could be in principle commercially viable, and quickly to provide data against which results from more detailed analytical models and experimental tests could later be compared.

This paper presents the preliminary analysis carried out in the first stage of the project, describing the assumptions and the details of the mathematical model regarding wave excitation, yacht's response, and power generation by the linear oscillator (section 2), and the results of simulations (section 3).

\section{Mathematical model}

\subsection{Wave spectra}

The main characteristic of sea waves is randomness. Indeed, by checking even a short time series, two characteristics arise: height and period of a wave are different from height and period of another wave. For this reason, the free surface elevation of sea waves is modelled as a stochastic process and is assumed to be a random, Gaussian, ergodic process in the time domain [15-18]; mathematically, the physical properties of sea waves are conveyed by the one-sided spectral density $S_{\zeta}(\omega)$ [17], where $\omega$ is the wave frequency ${ }^{1}$,

\footnotetext{
${ }^{1}$ In this paper, the term "frequency" will be used indifferently to identify both frequency $f$, measured in $\mathrm{Hz}$, or angular (circular) frequency $\omega$, measured in $\mathrm{rad} / \mathrm{s}$.
} 
which is related to the wave number by the dispersion relation, which, in deep water is $[15-18]$ :

$$
\omega^{2}=k g
$$

where $g$ is the acceleration of gravity. (It may be useful to recall that wave number and wave length are mutually dependent: $k=2 \pi / \lambda$ ).

The spectral density is correlated to the overall energy content of the sea state, therefore, statistical data that can be gleaned from the spectral density correspond to important parameters for the description of a sea state. Of particular importance is the 0 -th spectrum moment $m_{0}$, which is equivalent to the area under the wave spectrum curve, because many relevant physical properties directly derive from it. In particular, one of the most useful parameter to represent the sea state is the significant wave height, which is the average wave height (crest to trough) of the one-third largest waves, and for narrow band spectra it is given by [15-18]:

$$
h_{s}=4 \sqrt{m_{0}}
$$

The simulations that will be presented in the following sections were carried out taking into account statistical wave data for the Mediterranean Sea, with particular reference to measurements taken at Capo Linaro (Civitavecchia, Italy) by ISPRA (Istituto Superiore per la Protezione e la Ricerca Ambientale) from January $2^{\text {nd }}, 2004$, to September $12^{\text {th }}, 2006$.

In the case of random waves, it is possible to find a particular set of parameters that make the JONSWAP spectrum suitable to represent sea conditions in the location of interest.

The JONSWAP spectrum was developed from extensive field measurements in the context of the Joint North Sea Wave Project [15-18]. This formulation is suitable for wind-generated waves in fetch limited locations. The inputs are the wind speed and the fetch length. The mathematical formulation is given by the following equation:

$$
S_{\zeta}(\omega)=\alpha g^{2} \omega_{p}^{-5} \exp \left[-\frac{5}{4}\left(\frac{\omega}{\omega_{p}}\right)^{-4}\right] \exp \left\{\log \gamma \exp \left[-\frac{\left(\omega-\omega_{p}\right)^{2}}{2 \sigma^{2} \omega_{p}^{2}}\right]\right\}
$$

In the above equation, $\omega_{p}=2 \pi / T_{p}$ is the peak circular frequency, $\alpha$ is the Phillips' parameter given by $\alpha=0.0076(g x / \bar{U})^{-0.22}$, where $x$ is the fetch length and $\bar{U}$ the mean wind speed, and $\gamma$ is the peak-shape parameter. For 
practical applications, $\sigma$ can be assumed equal to 0.08 in the whole frequency domain.

Sea conditions at the chosen location are well represented by a bimodal spectrum [19, 20], with the relationship between peak period and significant wave height governed by two parameters $(\alpha$ and $\gamma)$ :

$$
T_{p}=f(\alpha, \gamma) \sqrt{h_{s} / g}
$$

The bimodal JONSWAP spectrum is completely defined when the significant wave height $h_{s}$ and parameters $\alpha$ and $\gamma$, along with the function $f(\alpha, \gamma)$, are specified.

In order to represent correctly sea conditions at Capo Linaro, values of $\alpha, \gamma, f(\alpha, \gamma)$ and $T_{p}$ are chosen according to table 1 (F. Arena, personal communications, 2012). The corresponding wave spectra are illustrated in fig. 1.

\subsection{Yacht model}

\subsubsection{Encounter frequency}

Due to its forward speed $V$, the wave spectrum for the ship is different than for a fixed observer. When studying the ship's response it is therefore necessary to take into account the frequency at which it actually encounters the waves (encounter frequency). The encounter frequency depends on wave velocity, ship speed and heading, i.e. the relative direction of the ship with respect to waves. Headings are denoted by means of the angle $\mu$, which is defined between the forward directions of wave and ship: thus for bow waves $\mu=\pi$, for transverse waves $\mu=\pi / 2$, and for aft waves $\mu=0$.

For seakeeping purposes, the assumption of deep water may be applied; in this case, taking into account the dispersion relation, eq. (1), the encounter frequency $\omega_{e}$ can be derived as:

$$
\omega_{e}=\omega-\frac{\omega^{2} V}{g} \cos \mu
$$

The spectral density must be modified according to the encounter frequency (it is practically a Doppler shift of the spectrum). Since the energy content of a spectrum must be the same for any observer, fixed or moving with the ship, the 0 -th momentum must be the same; as a result, the spectral 
density $S_{\zeta e}$ experienced by the ship is given by [18]:

$$
S_{\zeta e}\left(\omega_{e}\right)=\frac{S_{\zeta}(\omega)}{\left|1-2 \frac{\omega V}{g} \cos \mu\right|}
$$

\subsubsection{Response amplitude operators}

The frame of reference on a ship usually consists of a $X$-axis that coincides with the longitudinal axis of the ship and points from aft to bow, of a $Y$-axis that coincides with the transverse axis and points from port to starboard, and finally of a $Z$-axis for the vertical direction (positive upwards).

The six degrees of freedom for a ship are heave (vertical motion on the $Z$-axis), surge (longitudinal motion on the $X$-axis), sway (lateral motion on the $Y$-axis), yaw (oscillation around the vertical $Z$-axis), pitch (oscillation around the transverse $Y$-axis), roll (oscillation around the longitudinal $X$ axis). In this paper, only heave, pitch and roll are of interest, and they will be denoted respectively by variables $z, \vartheta$ and $\eta$.

The ship response to the wave excitation is usually described in terms of transfer functions (RAO, Response Amplitude Operator) [15-18], which give the normalised amplitude of the resulting ship's motion for a sinusoidal excitation of frequency $\omega_{e}$, the normalization factor being the wave amplitude $\zeta_{0}$ for linear motions and the wave slope $k \zeta_{0}=2 \pi \zeta_{0} / \lambda$ for angular motions.

$$
\operatorname{RAO}_{z}\left(\omega_{e}\right)=z_{0} / \zeta_{0}
$$

$$
\operatorname{RAO}_{\vartheta}\left(\omega_{e}\right)=\vartheta_{0} / k \zeta_{0}
$$

Obviously, equally important are the phase shifts $\varphi$ of each motion with respect to the wave excitation. With the knowledge of RAOs and phase shifts, it is possible to reconstruct heave, pitch and roll motions from a sinusoidal wave excitation. Therefore, based on the chosen frame of reference described at the beginning of this section, and taking into account that angular motions (pitch and roll) are usually small, vertical oscillations for any point on the ship may be calculated as follows:

$$
y(t) \cong z(t)-L \vartheta(t)+B \vartheta(t)
$$

where $L$ and $B$ are the longitudinal and lateral distance of any point on the ship from the center of gravity. 
Therefore, being the sum of harmonic motions (phasors), the vertical oscillation $y$ is also represented by a harmonic oscillation, and it is possible to define a RAO for the particular point of interest on the ship:

$$
\operatorname{RAO}_{y}\left(\omega_{e}\right)=y_{0} / \zeta_{0}
$$

In case of a random wave excitation, with the assumption that the response is a linear function of wave amplitude and applying the superposition principle, vertical motion can be reconstructed as:

$$
y(t)=\sum_{j=1}^{n} y_{0, j} \cos \left(\omega_{e, j} t+\varphi_{y, j}\right)
$$

where each oscillation amplitude $y_{0, j}$ is a function of frequency and amplitude of the $\mathrm{j}$-th harmonic, according to eq. (10).

Furthermore, it is possible to demonstrate that the ship's response spectral density is given by the product of the square of the RAO and the wave spectral density. Thus, for any point on the ship the spectral density associated to its wave-induced motion is:

$$
S_{y}\left(\omega_{e}\right)=\mathrm{RAO}_{y}^{2}\left(\omega_{e}\right) S_{\zeta e}\left(\omega_{e}\right)
$$

\subsection{Linear generator \\ 2.3.1. Equation of motion}

The linear generator used to recover energy from the wave-induced motions of the yacht is analysed and approximated in this paper as a simple linear mechanical oscillator [7], where the damping element represents a linear approximation of the effect of the electromagnetic force exerted by the generator as it provides a voltage difference proportional to the time derivative of the magnetic flux, and the spring represents the stiffness of the generator's support. It is assumed that the damping coefficient can be dynamically varied depending on sea conditions: this could be achieved in the final system by means of power electronics. Indeed, as the following sections will show clearly, it is very important to control the damping coefficient in order to achieve the best performance in terms of power generation under different operating conditions [10].

It is further assumed that the support can exert such a static force in order to balance the weight of the oscillating mass; it can be seen that mechanical springs cannot play such a role, because the resulting stiffness would be too 
high for the typical forcing frequencies. For example, if the spring were to counterbalance the weight with a limited elongation at rest $l=0.05 \mathrm{~m}$, the resulting natural frequency would be $\omega_{n}=\sqrt{g / l} \cong 2.2 \mathrm{~Hz}$, which is much larger than the forcing frequency of sea waves: this would make the system too stiff, i.e. the mass would move rigidly with the basement, with no relative motion between the two and, thus, no power extracted.

In the prototype that is being subjected to its first experimental testing, weight is balanced by the static forces generated by the interaction of coils and permanent magnets, placed in the moving part of the generator, with the "stator", which is simply an iron cylinder lathed with teeth and cavities (fig. 2). Other strategies could have been chosen to balance the weight of the oscillating mass, such as a pneumatic system: in this case, a proper selection of volumes could result in the desired (low) stiffness. However, the specific mechanism is not of particular interest for the analysis carried out in this paper, and in what follows it is simply assumed that static forces (weight and support) cancel each other.

The equation of motion can thus be written eliminating all static forces with reference to the relative position $s=x-y$ of the mass in a frame of reference moving with the basement:

$$
m \ddot{s}+c \dot{s}+K s=-m \ddot{y}
$$

which becomes the well-known second order ordinary differential equation for an oscillating body:

$$
\ddot{s}+2 \beta \omega_{n} \dot{s}+\omega_{n}^{2} s=-\ddot{y}
$$

with the introduction of the natural frequency of the oscillator:

$$
\omega_{n}=\sqrt{K / m}
$$

and of the damping ratio:

$$
\beta=\frac{c}{2 \sqrt{K m}}=\frac{c}{2 m \omega_{n}}
$$

\subsubsection{Electromagnetic damping}

A further important consideration is related to the damping coefficient, which can be written as:

$$
c=\frac{1}{R}\left[\frac{e(t)}{\dot{s}(t)}\right]^{2}
$$


208

209

$e(t)$ being the voltage generated according to Faraday's law $(e=-\mathrm{d} \Phi / \mathrm{d} t)$, and $R$ representing the external impedance; it is assumed that inductive impedance is negligible with respect to resistive impedance in the range of frequencies here considered [8].

In our case, there are three coils on the oscillating mass (fig. 2), each one generating a magnetic flux that can be seen, with a good degree of accuracy, as a sinusoidal function of relative position $s[12]$ :

$$
\Phi_{1}(s)=\Phi_{p} \cos \left(\frac{2 \pi}{w_{t}} s(t)\right)
$$

$$
\begin{aligned}
\Phi_{2}(s) & =-\Phi_{p} \cos \left(\frac{2 \pi}{w_{t}}\left(s(t)+\frac{1}{6} w_{t}\right)\right) \\
& =\Phi_{p} \cos \left(\frac{2 \pi}{w_{t}}\left(s(t)+\frac{2}{3} w_{t}\right)\right)
\end{aligned}
$$

where $\Phi_{p}$ is the maximum value of the flux and $w_{t}$ is the distance between the teeth on the stator (the second coil has opposite polarity with respect to the other coils, hence the minus sign).

From the time derivatives of each flux, we obtain the corresponding damping coefficients:

$$
c_{1}(t)=\frac{1}{R}\left[\frac{2 \pi \Phi_{p}}{w_{t}} \sin \left(\frac{2 \pi}{w_{t}} s(t)\right)\right]^{2}
$$

$$
c_{2}(t)=\frac{1}{R}\left[\frac{2 \pi \Phi_{p}}{w_{t}} \sin \left(\frac{2 \pi}{w_{t}}\left(s(t)+\frac{2}{3} w_{t}\right)\right)\right]^{2}
$$

$$
c_{3}(t)=\frac{1}{R}\left[\frac{2 \pi \Phi_{p}}{w_{t}} \sin \left(\frac{2 \pi}{w_{t}}\left(s(t)+\frac{2}{6} w_{t}\right)\right)\right]^{2}
$$

The total force exerted is given by the sum of the forces generated by each coil, so that the overall damping coefficient is the sum of each coil's damping coefficient: since each $c_{j}$ has the same sinusoidal pattern, only with a phase lag of $w_{t} / 6$, the sum is a constant:

$$
c(t)=\sum_{j=1}^{3} c_{j}(t)=\frac{3}{2}\left(\frac{2 \pi}{w_{t}}\right)^{2} \frac{\Phi_{p}^{2}}{R}
$$


This reasoning shows that representing the electromagnetic force with a linear dependency on velocity $\dot{s}$ is indeed a good approximation, as long as the magnetic flux can be represented accurately enough as a sinusoidal function of relative position $s$.

\subsubsection{Power generation}

The steady-state response of the linear mechanical system to a harmonic forcing is itself harmonic, with an amplitude $s_{0}$ given by:

$$
\frac{s_{0}}{y_{0}}=\operatorname{RAO}_{s}\left(\beta, \omega_{n}, \omega_{e}\right)=\frac{n^{2}}{\sqrt{\left(1-n^{2}\right)^{2}+(2 \beta n)^{2}}}
$$

where $n$ is the ratio of forcing to natural frequency:

$$
n=\omega_{e} / \omega_{n}
$$

In this model, electromagnetic damping provides the mean to extract energy from the wave excitation; thus, it is interesting to identify optimal values for the damping coefficient $c$ in order to extract the maximum power. The power absorbed is given by:

$$
P(t)=c \dot{s}^{2}
$$

and its average value over one cycle (which will be indicated as $\Pi$ ) is:

$$
\Pi=\frac{1}{T} \int_{0}^{T} P(t) \mathrm{d} t=\frac{1}{2} c \omega_{e}^{2} s_{0}^{2}
$$

This equation, taking into account eqs. (16) and (25), becomes:

$$
\Pi=m \omega_{e}^{3} y_{0}^{2} \frac{n^{3} \beta}{\left(1-n^{2}\right)^{2}+(2 \beta n)^{2}}
$$

Since the oscillations are constrained by the size of the generator, two different scenarios must be considered. In the first one, let us imagine that the undamped oscillations do not reach the maximum range allowed $s_{\max }$ : in this case, increasing the damping coefficient from 0 initially yields higher values of $\Pi$ even though $s_{0}$ decreases according to eq. (25), until a maximum for $\Pi$ is reached, beyond which it decreases. The optimum value of $\beta$ can thus be found when $\mathrm{d} \Pi / \mathrm{d} \beta=0$ :

$$
\beta_{\mathrm{opt}}=\frac{1}{2} \frac{\left|1-n^{2}\right|}{n}
$$


244

245

with corresponding optimum damping coefficient, maximum average power and oscillation amplitude given by the following equations:

$$
c_{\mathrm{opt}}=m \omega_{e}\left|1-\frac{1}{n^{2}}\right|
$$

$$
\Pi_{\max }=\frac{1}{4} m \omega_{e}^{3} y_{0}^{2}\left|1-\frac{1}{n^{2}}\right|^{-1}
$$

$$
s_{0}=y_{0} \frac{1}{\sqrt{2}}\left|1-\frac{1}{n^{2}}\right|^{-1}
$$

In the second case, the undamped oscillations would be larger than the maximum allowed range $s_{\max }$ : then it is possible to extract more power by increasing the damping coefficient while the oscillation amplitude $s_{0}$ remains at its maximum permissible level $s_{\max }$. It is possible to show that the maximum power is obtained when the damping coefficient is such that the oscillation given by eq. (25) is exactly equal to the maximum stroke $\left(s_{0}=s_{\max }\right)$ :

$$
\beta_{\mathrm{opt}}=\frac{n}{2} \sqrt{\left(\frac{y_{0}}{s_{\max }}\right)^{2}-\left(1-\frac{1}{n^{2}}\right)^{2}}
$$

$$
c_{\mathrm{opt}}=m \omega \sqrt{\left(\frac{y_{0}}{s_{\max }}\right)^{2}-\left(1-\frac{1}{n^{2}}\right)^{2}}
$$

and the corresponding maximum average power is:

$$
\Pi_{\max }=\frac{1}{2} m \omega_{e}^{3} s_{\max }^{2} \sqrt{\left(\frac{y_{0}}{s_{\max }}\right)^{2}-\left(1-\frac{1}{n^{2}}\right)^{2}}
$$

If the mechanical system is "tuned" to the forcing wave excitation $(n \cong 1)$, then the above expression can be simplified as follows:

$$
\Pi_{\max } \cong \frac{1}{2} m \omega_{e}^{3} s_{\max } y_{0}
$$

It is possible to find out which wave excitations make the system reach its maximum stroke $s_{\max }$ by setting the oscillation amplitude given by eq. (33) equal to $s_{\max }$, yielding:

$$
\frac{y_{0}}{s_{\max }}=\sqrt{2}\left|1-\frac{1}{n^{2}}\right|
$$


Thus, for wave amplitudes originating boat oscillations lower than the limit set by the above equation, the system oscillates "freely" and eqs. (3133) apply, while for higher waves more damping, and thus more power, is available, in order to constrain the system within the maximum stroke allowed, and eqs. (35-36) apply.

It is worth to point out that in both cases the optimum value for the damping coefficient is directly proportional to the oscillator's mass and to the forcing frequency: the average power absorbed is therefore proportional to the mass $m$ and to the third power of forcing frequency $\left(\omega^{3}\right)$, as shown by eqs. (32) and (36).

In particular, the linear dependence on the oscillating mass $m$ is, on the one hand, almost obvious because energy recovery depends on inertia and kinetic energy, but on the other hand it is an important property to be taken into account because it allows to design, test and prototype modular systems of relatively low mass, with the overall power extracted given by the sum of power available from different modules. For this reason, the results discussed in section 3 will be given with reference to a unit mass $m=1 \mathrm{~kg}$.

The frequency response of the harmonic oscillator can also be used when the external forcing is not harmonic (as in the case of a real wave excitation): if $S_{y}(\omega)$ is the spectral density associated to the vertical oscillations of a particular point of interest, which can be evaluated from the wave spectral density by means of eq. (12), then the spectral density associated to the relative motion $s$ of a linear system such as the one described in the previous section is given by:

$$
S_{s}\left(\beta, \omega_{n}, \omega_{e}\right)=\operatorname{RAO}_{s}\left(\beta, \omega_{n}, \omega_{e}\right)^{2} S_{y}\left(\omega_{e}\right)
$$

In the following considerations the dependence of relative motion and its spectrum on natural frequency $\omega_{n}$ will be implicitly assumed, so that $S_{s}\left(\beta, \omega_{e}\right) \equiv S_{s}\left(\beta, \omega_{n}, \omega_{e}\right)$.

The spectrum of relative motion allows the evaluation of the significant oscillation amplitude as follows:

$$
s_{0 s}(\beta)=2 \sqrt{m_{0 s}}=2 \sqrt{\int_{0}^{\infty} S_{s}\left(\beta, \omega_{e}\right) \mathrm{d} \omega_{e}}
$$

Since power generation depends on the square of the generator's velocity (eq. 27), the spectral density of relative velocity $\dot{s}$ must be introduced. This 
is simply given by:

$$
S_{\dot{s}}\left(\beta, \omega_{e}\right)=\omega_{e}^{2} S_{s}\left(\beta, \omega_{e}\right)=\omega_{e}^{2} \operatorname{RAO}_{s}\left(\beta, \omega_{e}\right)^{2} S_{y}\left(\omega_{e}\right)
$$

The zero-th momentum of the velocity spectrum gives velocity's root mean square (RMS), which is related to the average power generation:

$$
\dot{s}_{\mathrm{RMS}}(\beta)=\sqrt{m_{0 \dot{s}}}=\sqrt{\int_{0}^{\infty} S_{\dot{s}}\left(\beta, \omega_{e}\right) \mathrm{d} \omega_{e}}
$$

$$
\Pi_{\mathrm{RMS}}(\beta)=\frac{c}{m} \dot{s}_{\mathrm{RMS}}^{2}(\beta)=2 \beta \omega_{n} \dot{s}_{\mathrm{RMS}}^{2}(\beta)
$$

As in the case of sinusoidal waves, for a given natural frequency the generator's motion depends on the choice of the damping coefficient $\beta$, for which an optimum value is found by maximizing power output (eq. 43) with the constraint that the significant oscillation amplitude be lower than the maximum allowed range $s_{\max }$ (for this non-linear optimization procedure the MATLAB ${ }^{\circledR}$ function fmincon has been used):

$$
\left.\frac{\mathrm{d} \Pi_{\mathrm{RMS}}}{\mathrm{d} \beta}\right|_{\beta_{\mathrm{opt}}}=0 \quad \text { with } \quad s_{0 s}\left(\beta_{\mathrm{opt}}\right) \leq s_{\max }
$$

It must be observed that this optimization procedure is based on a statistical evaluation of the oscillation amplitude, and therefore the results are approximated, because the spectrum calculated in this way contains also oscillations higher than the constraint $s_{\max }$ : as a consequence, the actual power generation corresponding to this spectrum is somewhat lower than the value given by eq. 43 because a fraction of the energy contained in the largest oscillations is lost as the moving mass hits the shock absorbers. This process is highly non-linear, and this is the reason why it is not included in the linearised frequency-domain model here presented.

\section{Results}

\subsection{Yacht response}

The foundation of the commercial software package Seakeeper, which was used to carry out the computation of the yacht's motions under different wave conditions, is the linear strip theory [21], which is used to calculate the 
coupled heave and pitch response of the vessel; the roll response is calculated using linear roll damping theory [22].

The main purpose of the kinematic model presented is to provide reasonable data about the response of a generic yacht to different sea conditions, in order to have reliable information on the motion which the SEAKERS device is subjected to. Since the project does not address a particular yacht model, nor even a specific size of boat, there was no point in developing a focused in-house software: hence the choice of adopting a commercial software that has a proven record of reliability, using it to simulate the response of a yacht of adequate length included in the extensive library provided.

The yacht's model used in the numerical simulations is one of the library models that can be found in Seakeeper's library, since it has geometric and mass properties comparable to those of commercial sail yachts of interest for the SEAKERS project.

The most relevant hydrostatic properties [16] of this yacht are given in table 2, while the shape of the hull (with the indication of the waterline) and the generator's position are represented in figs. 3 and 4 . The generator considered in the simulations presented is placed astern on the longitudinal axis $(B=0)$ at a distance $L=-4.20 \mathrm{~m}$ from the center of gravity, i.e. close to the helm.

Sectional hydrodynamic masses, damping coefficients, and all other data needed in the context of the strip theory [22] have been evaluated by means of 21 two-dimensional sections (conformal mappings) approximating hull's geometry and features.

As illustrated in section 2.2, the yacht's response is defined by means of Response Amplitude Operators (RAO) and phase shifts, which have been obtained by means of the Seakeeper software, and provide the basis for the evaluation of the response at a particular point on the ship (eqs. 10 and 11). Figure 5 shows values of RAO at the location of the linear generator for three different speeds $(V=[3,6,9] \mathrm{knt})$ and two headings $(\mu=[90,135] \mathrm{deg})$.

The response to random waves is illustrated in figs. 6 and 7 in terms of spectral density of the vertical oscillations, as defined by eq. (12). The significant oscillation amplitudes are obtained from these spectra in the same way as the significant wave amplitude is calculated from the wave spectrum [17]:

$$
y_{0 s}=2 \sqrt{m_{0 y}}=2 \sqrt{\int_{0}^{\infty} S_{y}\left(\omega_{e}\right) \mathrm{d} \omega_{e}}
$$


Values of significant vertical oscillation amplitudes, corresponding to the spectral densities of figs. 6 and 7 , are given in table 3 .

\subsection{Linear generator}

In this section, results obtained with the mechanical model of the linear generator subject to random wave excitations, for the same conditions of speed and heading considered in section 3.1, are discussed.

Figures 8 and 9 show the energy spectra associated to the generator's relative motion and RMS power generation for different speeds and directions. Power generation values are also given in table 4 , while table 5 reports significant oscillation amplitudes.

The main target for the SEAKERS project is a sail yacht of length ranging from 10 to $14 \mathrm{~m}$, and in this class of yachts the maximum height available for a possible installation of the device is just above $1 \mathrm{~m}$ : therefore, the maximum stroke taken into consideration in the simulations is $s_{\max }=0.5 \mathrm{~m}$. The natural frequency $\omega_{n}$ is taken as $0.40 \mathrm{~Hz}$, in order to make the mechanical system almost resonant with most sea conditions that may be encountered (a parametric analysis has been carried out in order to find an optimum value).

From table 5 it is possible to see that the generator's oscillation is almost always limited to the maximum range $s_{\max }$, even when the vertical oscillation of the yacht is low (table 3): this happens thanks to almost-resonant operating conditions and to variable damping coefficients. In order to analyse the results, it is thus useful to consider the simplified equation for average power generation, eq. (37), which shows that, if the mechanical system is "tuned" to the forcing wave $(n \cong 1)$, the average power is proportional to the third power of the encounter frequency, and to the product of boat's vertical oscillation $y_{0}$ and maximum range $s_{\max }$.

The spectral densities represented in figs. 8 and 9 may seem surprising at first, because the energy content appears to be almost the same for different wave heights, even though the corresponding vertical motions of the yacht are quite different (figs. 6-7 and table 3). Obviously, this just reflects the constraints on the oscillator's vertical motion, limited to $\pm s_{\max }$, but it does not mean that power generation is the same for different forcing conditions: as tables 4 and 6 show, more power is available with higher waves because a higher damping coefficient can be used. This underscores the relevance of a properly configured control system, able to identify ongoing sea conditions and adjust the electromagnetic properties of the system accordingly. 
More specifically, an increase in significant wave height gives rise to two opposite effects on power generation: on the one hand it increases due to its dependence on $y_{0}$, but on the other hand the spectral density shifts towards lower frequencies (fig. 1), resulting in a decrease in power generation. Clearly, this gives rise to a maximum power generation for a particular sea state, that under the assumptions taken into account in this paper correspond to a significant wave height of $1.5 \mathrm{~m}$, as figs. 8 and 9 , along with table 4 , show.

In other words, even with high values of significant wave height, which correspond to rather low values of peak frequencies (table 1), if the full spectrum is taken into account significant contributions to the excitation can be found also at relatively high frequencies, and these contributions increase average velocities and, consequently, power generation. Nonetheless, it is still possible to find that waves higher than a certain threshold actually decrease power output, because in this case significant contributions would indeed be found only at low frequencies: that is why power generation is found to be lower for $2.0 \mathrm{~m}$ wave height than for $1.5 \mathrm{~m}$.

Tables 6 and 7 report values of optimum damping coefficients and damping ratios as defined by the optimization procedure, eq. (44).

\section{Discussion and conclusions}

This paper has provided an overview of the results obtained with the kinematic and mechanical models of the yacht's response to different wave excitations, and of the linear generator taken as a simple mechanical linear system which extracts power from the wave-induced motion by means of an ideal linear electromagnetic damping.

These preliminary evaluations, even though based on a rather simplified model of the generator, have produced some important insights on system dynamics and on the range of values to be assigned to several significant parameters, such as mechanical stiffness and damping ratio.

In particular, given the particular range of forcing frequencies, the mechanical stiffness must be chosen so as to obtain a natural frequency within the range of most forcing frequencies: a value of $0.40 \mathrm{~Hz}$ has been considered in this paper, with a resulting stiffness-to-mass ratio around $6.3 \mathrm{~N} /(\mathrm{m} \mathrm{kg})$.

The choice of damping ratios is based on the maximisation of power output for a given natural frequency for different wave excitation conditions. Values of optimum damping coefficients relative to mass have been found in 
the range $1.3-3.9 \mathrm{~N} \mathrm{~s} /(\mathrm{m} \mathrm{kg})$, and this result will be useful in the definition of the electric circuit's physical parameters.

Power generation of up to $2.1 \mathrm{~W} / \mathrm{kg}$ has been obtained in the most favourable sea conditions, and anyway values higher than $0.5 \mathrm{~W} / \mathrm{kg}$ are available in most cases, which represent an interesting result for this particular application. Indeed, a total weight for the SEAKERS device of up to $200 \mathrm{~kg}$ can be considered acceptable on sail yachts with length in the range $12-14 \mathrm{~m}$, especially in the case of a first equipment (as opposed to retrofitting an already existing yacht, because in this case many more design constraints should be addressed). With an optimized design, it is conceivable from preliminary evaluations that up to $50 \%$ of this weight $(100 \mathrm{~kg})$ could be allocated to the oscillating masses; in this case, an average power generation of almost $100 \mathrm{~W}$ is feasible, which could make possible to recover at least $1 \mathrm{kWh}$ at the end of a day-long cruise. This amount of energy generation could indeed be interesting for this particular application.

Obviously, the issue related to the influence of this moving mass on sailing performance should be addressed: but specific calculations, which have not been reported here for brevity's sake, show that the inertial forces generated by the mass' motion are at least two orders of magnitude lower than the forces exerted by the sea on the boat. After all, this must be the case because the energy absorbed by the linear system is but a small fraction of the total energy of the incoming waves. Therefore, in all probability the impact of the added mass due to the generator on sailing performance can be safely deemed negligible.

It should be observed that the concept of "power availability" (or "availability factor") routinely used to appreciate the performance of a system based on renewable energy, is much less useful for this particular application, because the final goal is not the generation of electricity per se on a continuous basis but, rather, only on the particular occasion when the yacht is used for a cruise. An availability factor should therefore be considered only with reference to a single cruise, and it would take into account the time frequency of encountered wave height during a typical cruise of a sailing boat, because this is the main parameter influencing average power output. However, it is rather difficult to make any prediction about this probability, other than saying that sail cruises are most common when wind (and, consequently, wave) conditions are not extreme (i.e. with moderate winds and waves, while it is safe to assume that calm or stormy seas are avoided); furthermore, a leisure cruise usually requires stable weather conditions. In the end, it is reasonable 
to infer that in most cases sea conditions encountered on a leisure cruise are reasonably constant and marked by a significant wave height within the range 0.5-1.5 $\mathrm{m}$. Under these assumptions, average power generation is expected to be almost constant during the whole cruise.

\section{Acknowledgments}

The research leading to these results has received funding from the European Union Seventh Framework Programme (FP7/2007-2011) under grant agreement n. 262591. The authors would also like to acknowledge the invaluable effort provided by all the staff of Labor srl, the projects' coordinator, as well as the contribution of the SMEs and RTD performers involved in the project.

\section{References}

[1] de O. Falcão A. Wave energy utilization: A review of the technologies. Renewable and Sustainable Energy Reviews 2010;14:899-918.

[2] Langhamer O, Haikonen K, Sundberg J. Wave power-sustainable energy or environmentally costly? A review with special emphasis on linear wave energy converters. Renewable and Sustainable Energy Reviews 2010;14:1329-35.

[3] Elwood D, Yim S, Prudell J, Stillinger C, von Jouanne A, Brekken T, et al. Design, construction, and ocean testing of a taut-moored dualbody wave energy converter with a linear generator power take-off. Renewable Energy 2010;35:348-54.

[4] Huang SR, Chen HT, Chung CH, Chu CY, Li GC, Wu CC. Multivariable direct-drive linear generators for wave energy. Applied Energy 2012;100:112-7.

[5] Babarit A, Hals J, Muliawan M, Kurniawan A, Moan T, Krokstad J. Numerical benchmarking study of a selection of wave energy converters. Renewable Energy 2012;41:44-63.

[6] Ahn K, Truong D, Tien H, Yoon J. An innovative design of wave energy converter. Renewable Energy 2012;42:186-94. 
[7] Stephen N. On energy harvesting from ambient vibration. Journal of Sound and Vibration 2006;293:409-25.

[8] El-hami M, Glynne-Jones P, White N, Hill M, Beeby S, James E, et al. Design and fabrication of a new vibration-based electromechanical power generator. Sensors and Actuators 2001;92:335-42.

[9] Ivanova I, Bernhoff H, Ågren O, Leijon M. Simulated generator for wave energy extraction in deep water. Ocean Engineering 2005;32:1664-78.

[10] Eriksson M, Isberg J, Leijon M. Hydrodynamic modelling of a direct drive wave energy converter. International Journal of Engineering Science 2005;43:1377-87.

[11] Leijon M, Danielsson O, Eriksson M, Thorburn K, Bernhoff H, Isberg $\mathrm{J}$, et al. An electrical approach to wave energy conversion. Renewable Energy 2006;31:1309-19.

[12] Thorburn K, Leijon M. Farm size comparison with analytical model of linear generator wave energy converters. Ocean Engineering 2007;34:908-16.

[13] Castellucci V, Waters R, Eriksson M, Leijon M. Tidal effect compensation system for point absorbing wave energy converters. Renewable Energy 2013;51:247-54.

[14] Beeby S, Tudor M, White N. Energy harvesting vibration sources for microsystems applications. Measurement Science and Technology 2005;17:175-95.

[15] Bertram V. Practical ship hydrodynamics. Oxford, UK: ButterworthHeinemann; 1999.

[16] Biran A. Ship hydrostatics and stability. Oxford, UK: ButterworthHeinemann; 2003.

[17] Jensen J. Load and global response of ships. Oxford, UK: Elsevier; 2001.

[18] Rawson K, Tupper E. Basic ship theory. Oxford, UK: ButterworthHeinemann; 2001. 
518

519

520

521

522

523

524

525

526

[19] Soares CG. Representation of double-peaked sea wave spectra. Ocean Engineering 1984;11:185-207.

[20] Arena F, Soares CG. Nonlinear high wave groups in bimodal sea states. Journal of Waterway, Port, Coastal, and Ocean Engineering 2009;135:69-79.

[21] Salvesen N, Tuck O, Faltinsen O. Ship motions and sea loads. Transactions, Society of Naval Architects and Marine Engineers 1970;78:250-87.

[22] Seakeeper v. 16 - User Manual. Formation Design Systems Pty Ltd; 2011. 


\section{List of Figures}

1 Wave spectra representing sea conditions at Capo Linaro near Civitavecchia, Italy. . . . . . . . . . . . . . . . . . . . . . . . . 22

2 Representation of the linear generator: the oscillating mass (on the outside) carries coils and permanent magnets. . . . . . 23

3 Profile view of the yacht's hull. . . . . . . . . . . . . 24

4 Plan view of the yacht's hull. . . . . . . . . . . . 25

5 Response Amplitude Operator at the location of the linear generator. . . . . . . . . . . . . 26 26

6 Spectral density of the yacht's vertical oscillation at the gen-

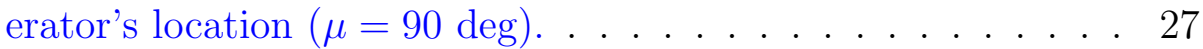

7 Spectral density of the yacht's vertical oscillation at the gen-

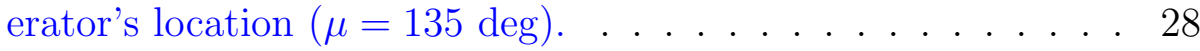

8 Spectral density of the generator's vertical oscillation and av-

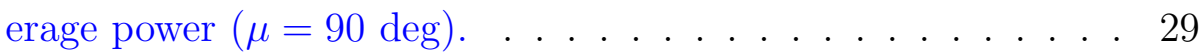

9 Spectral density of the generator's vertical oscillation and av-

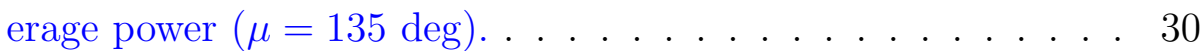

\section{List of Tables}

1 Parameters used to represent random sea waves at Capo Linaro near Civitavecchia, Italy. . . . . . . . . . . . . . . . 31

2 Hydrostatic properties. . . . . . . . . . . . . . . 32

3 Yacht's significant vertical oscillation amplitudes at the generator's location. . . . . . . . . . . . . . . . . . 33

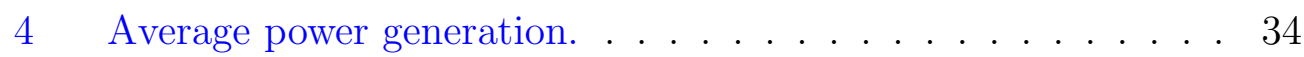

5 Generator significant oscillation amplitudes. . . . . . . . . 35

6 Optimum damping coefficients for random wave excitation. . . 36

7 Optimum damping ratios for random wave excitation. . . . . . 37 


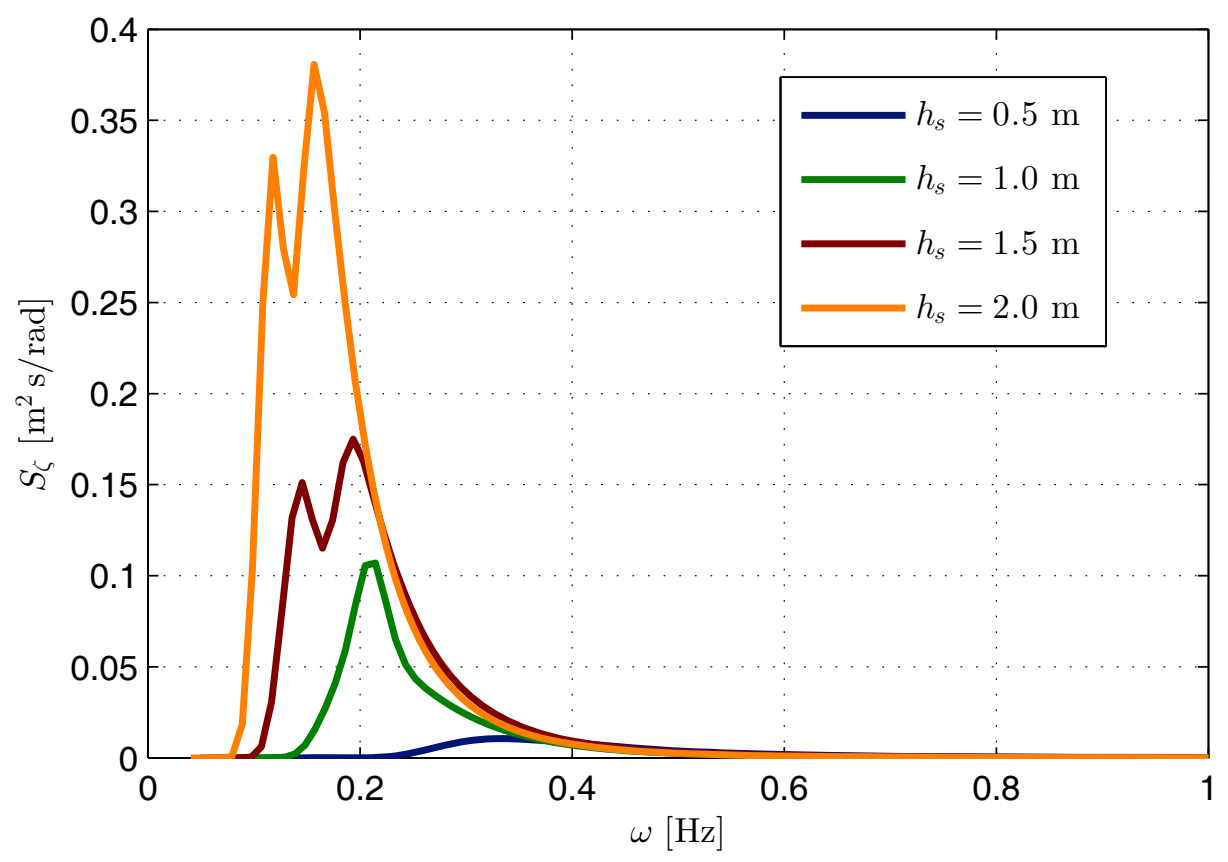

554

Figure 1: Wave spectra representing sea conditions at Capo Linaro near Civitavecchia, Italy. 


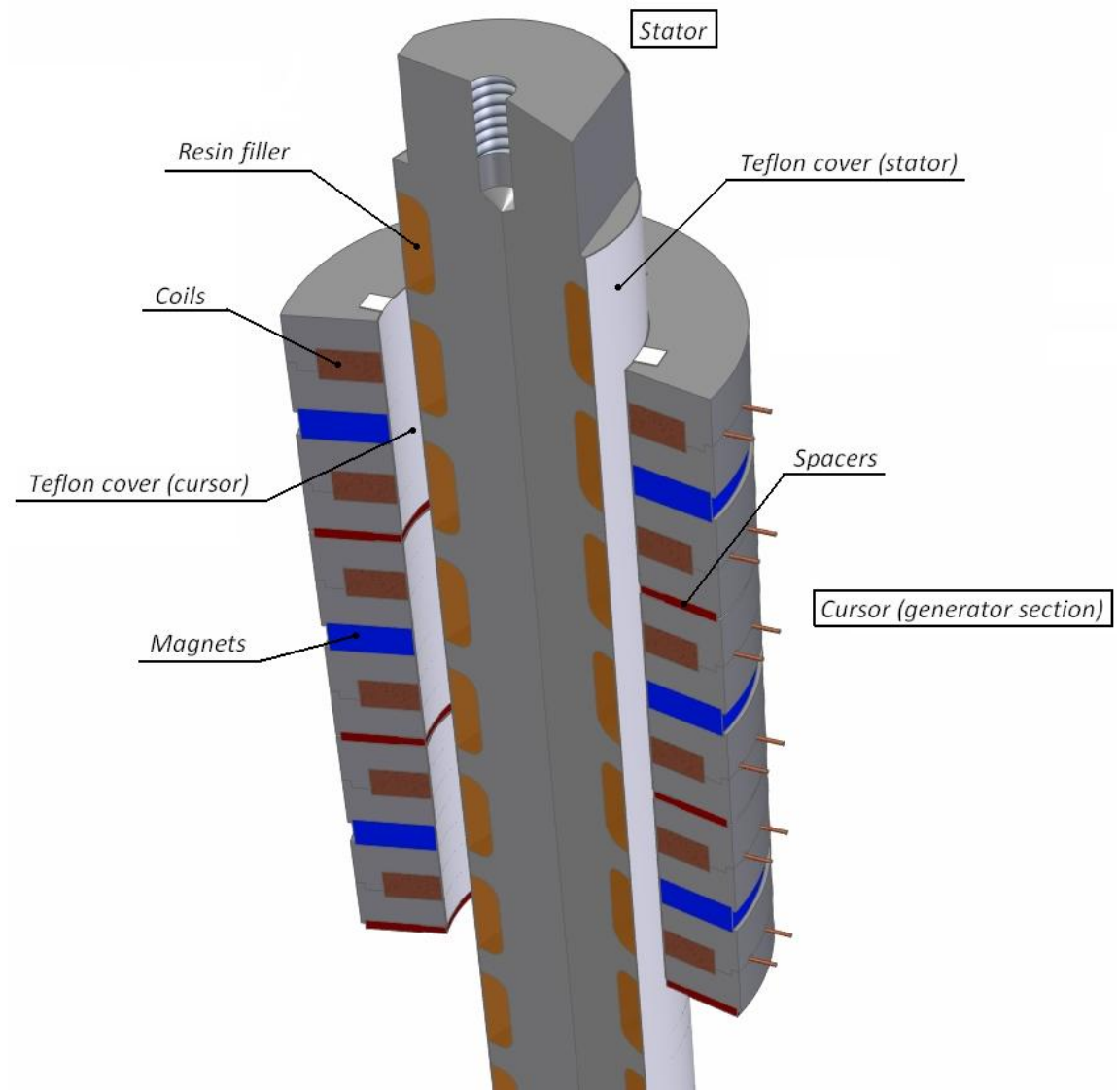

Figure 2: Representation of the linear generator: the oscillating mass (on the outside) carries coils and permanent magnets. 


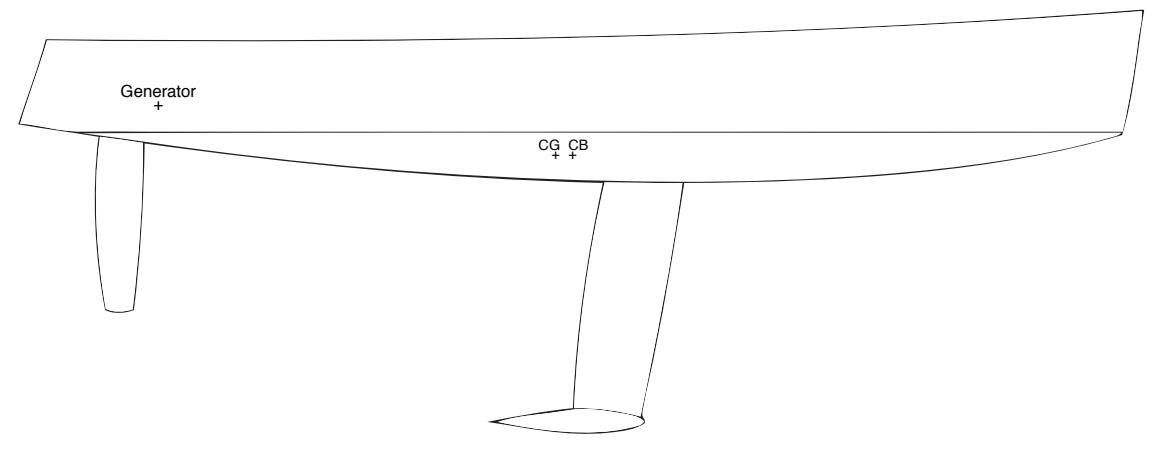

556

Figure 3: Profile view of the yacht's hull. 


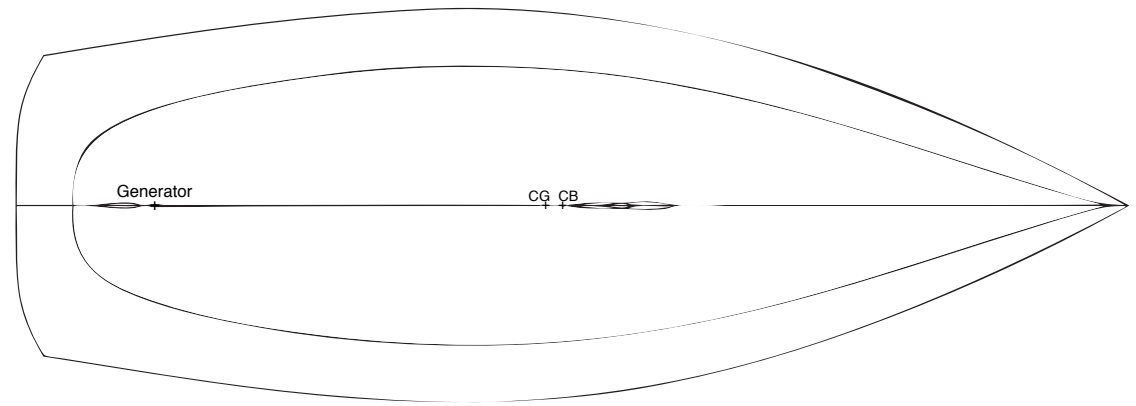

557

Figure 4: Plan view of the yacht's hull. 

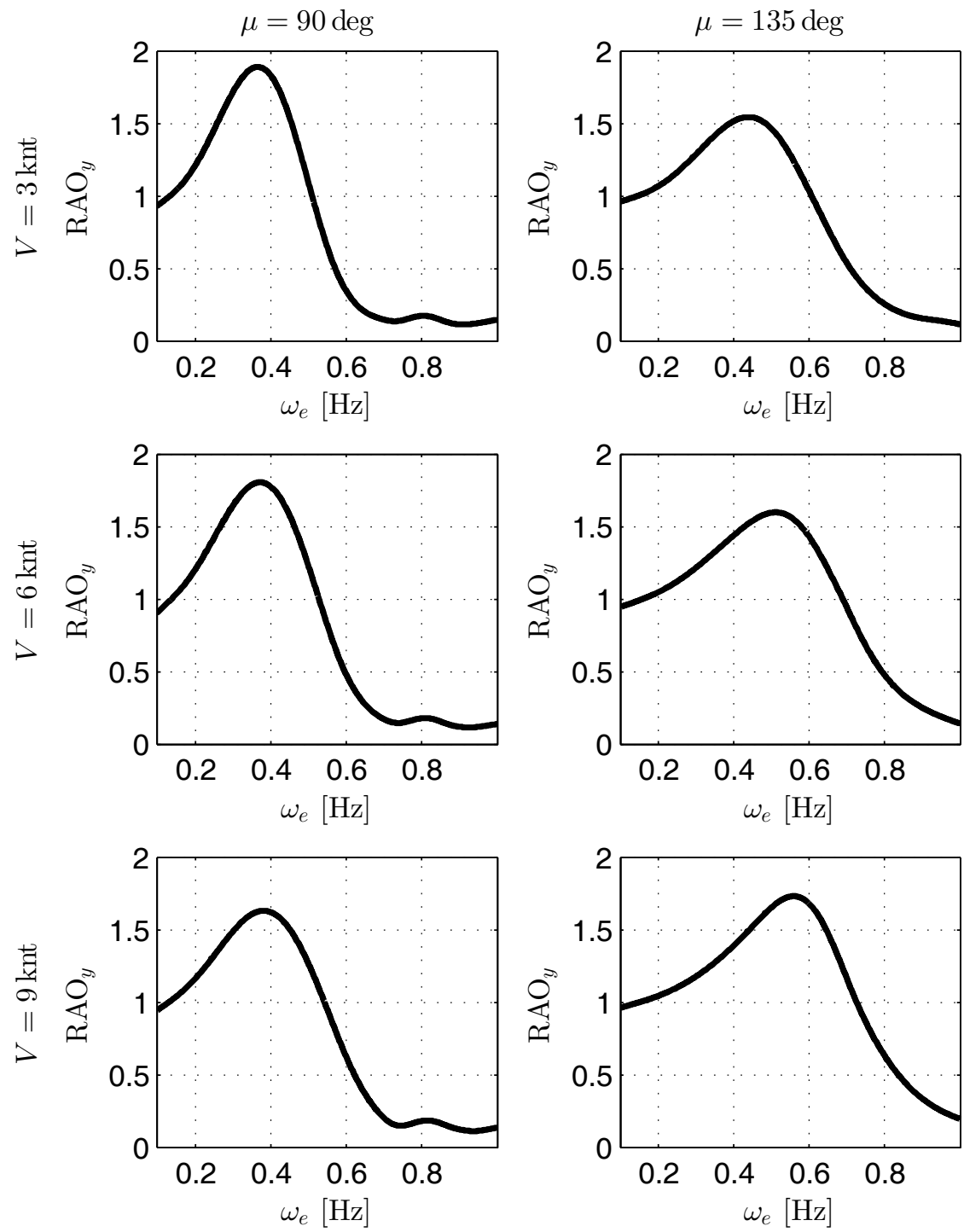

558

Figure 5: Response Amplitude Operator at the location of the linear generator. 

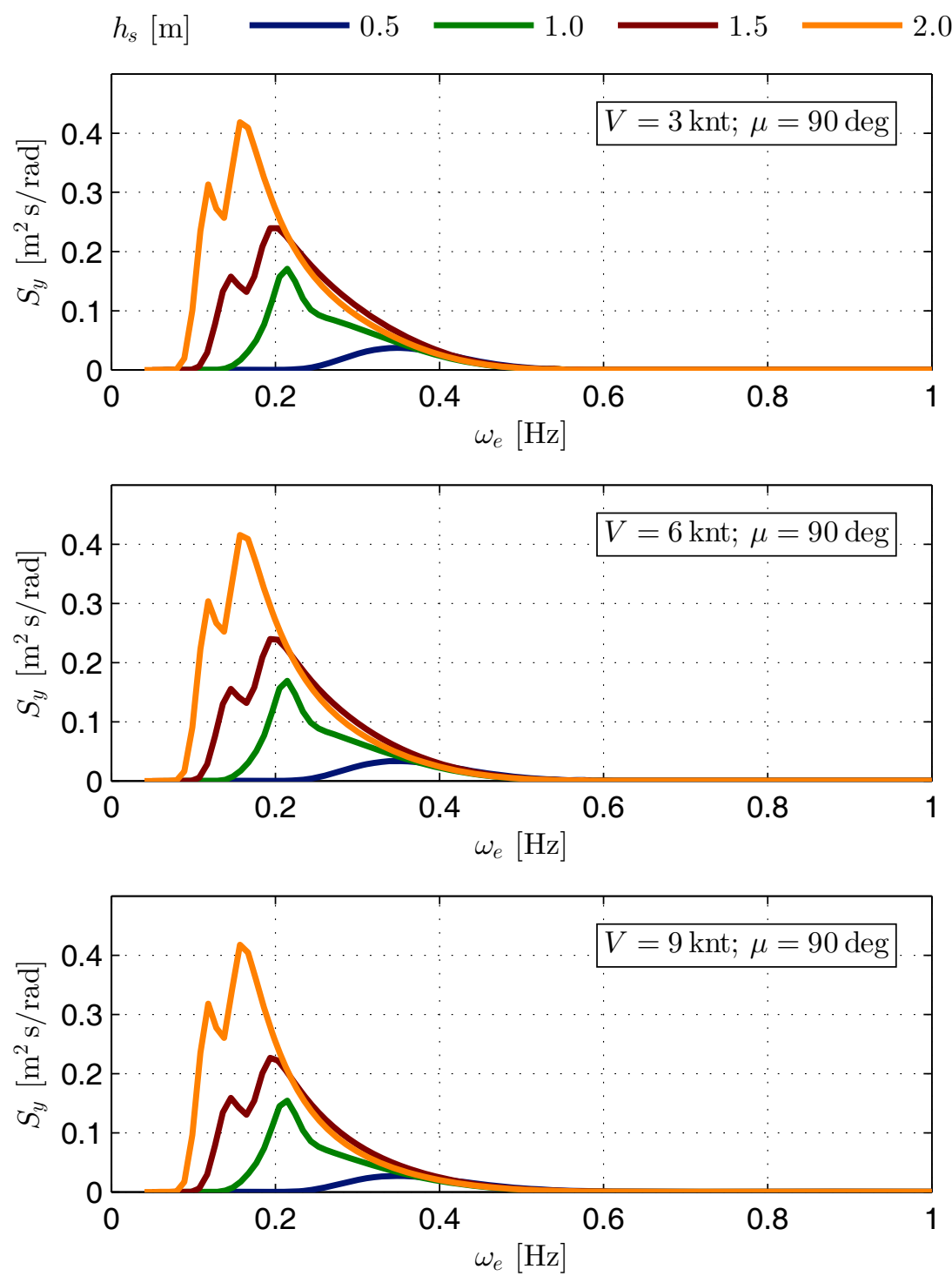

559

Figure 6: Spectral density of the yacht's vertical oscillation at the generator's location $(\mu=90 \mathrm{deg})$. 

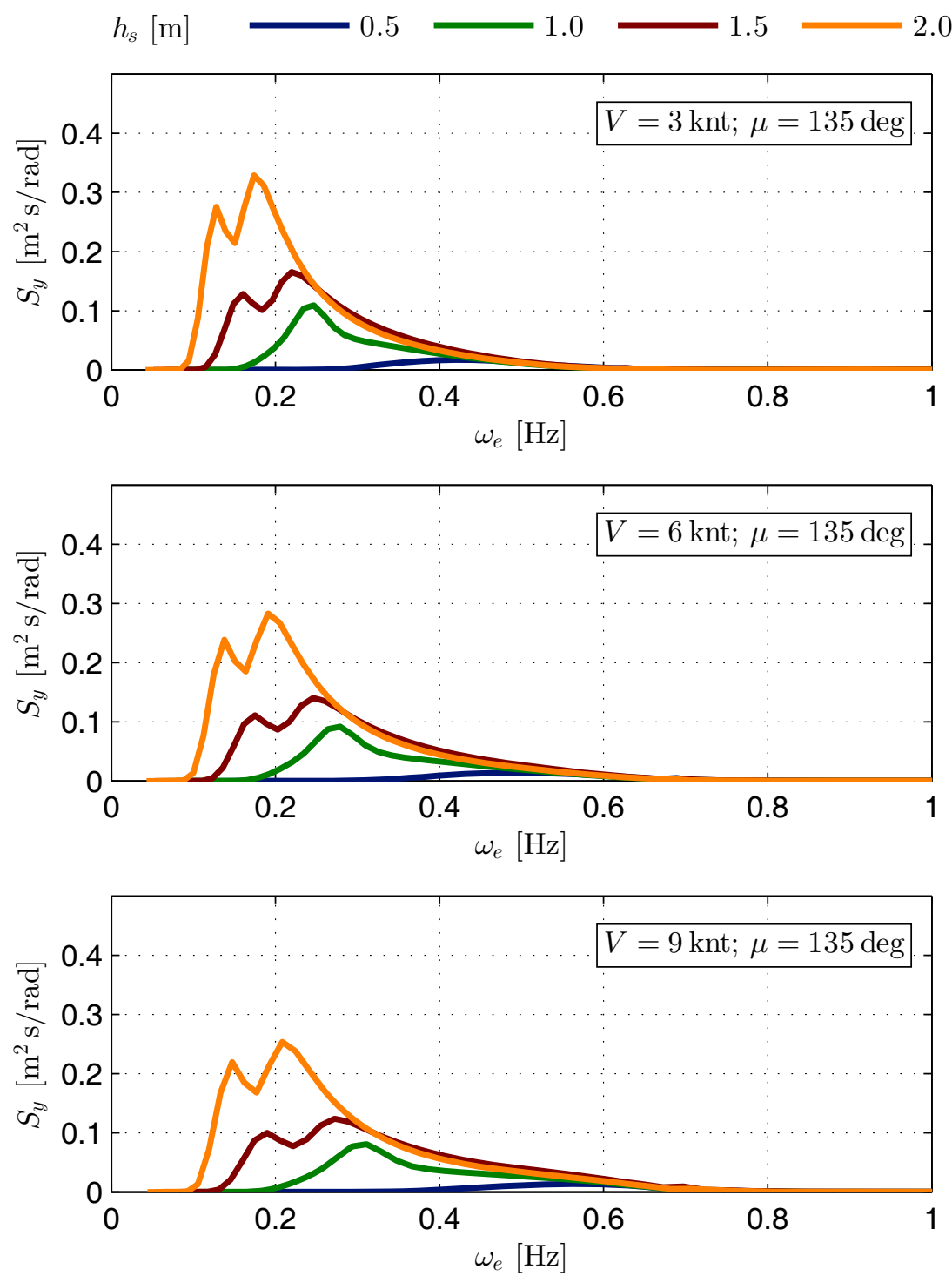

560

Figure 7: Spectral density of the yacht's vertical oscillation at the generator's location $(\mu=135 \mathrm{deg})$. 

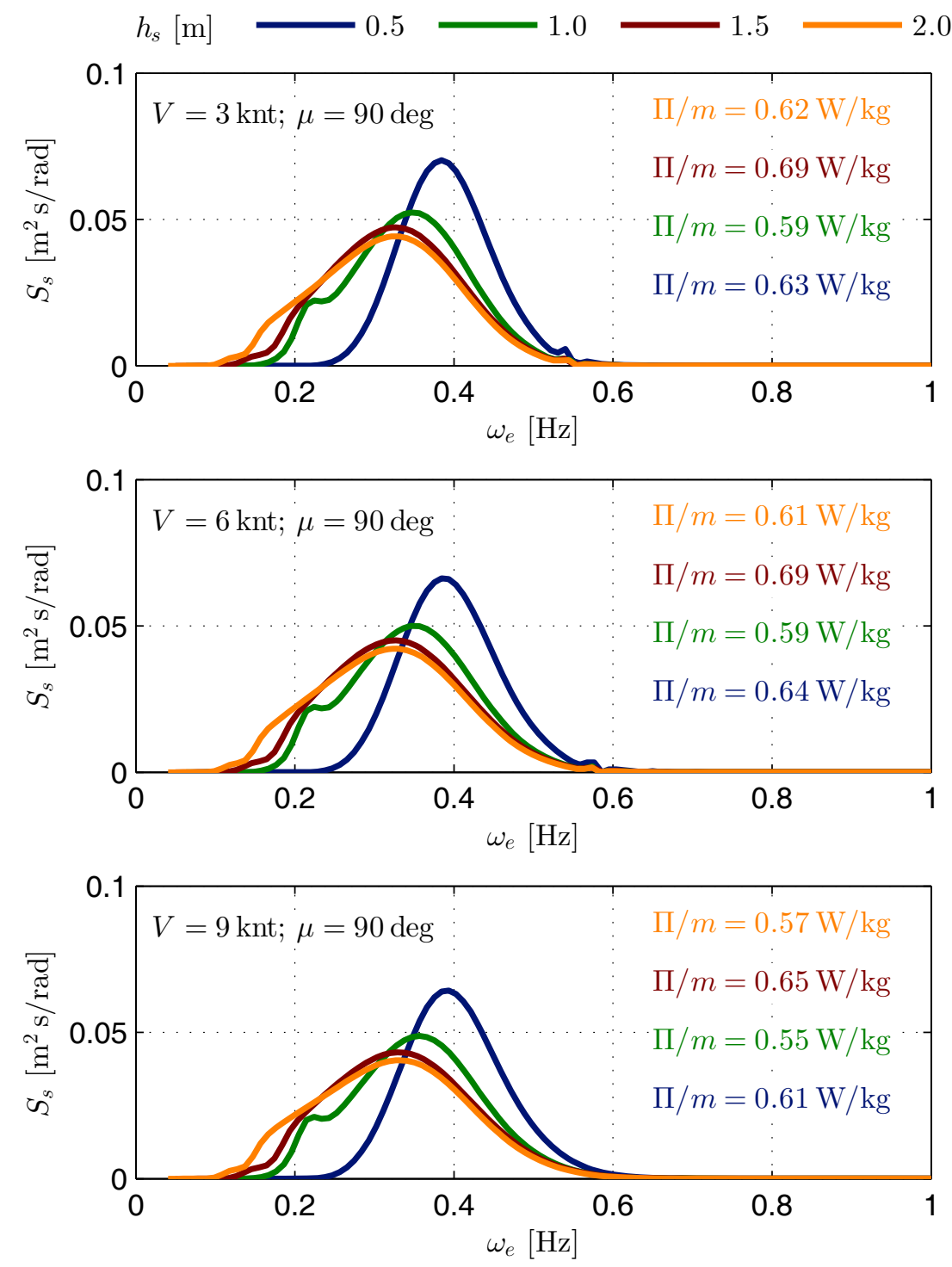

561

Figure 8: Spectral density of the generator's vertical oscillation and average power $(\mu=$ $90 \mathrm{deg})$. 

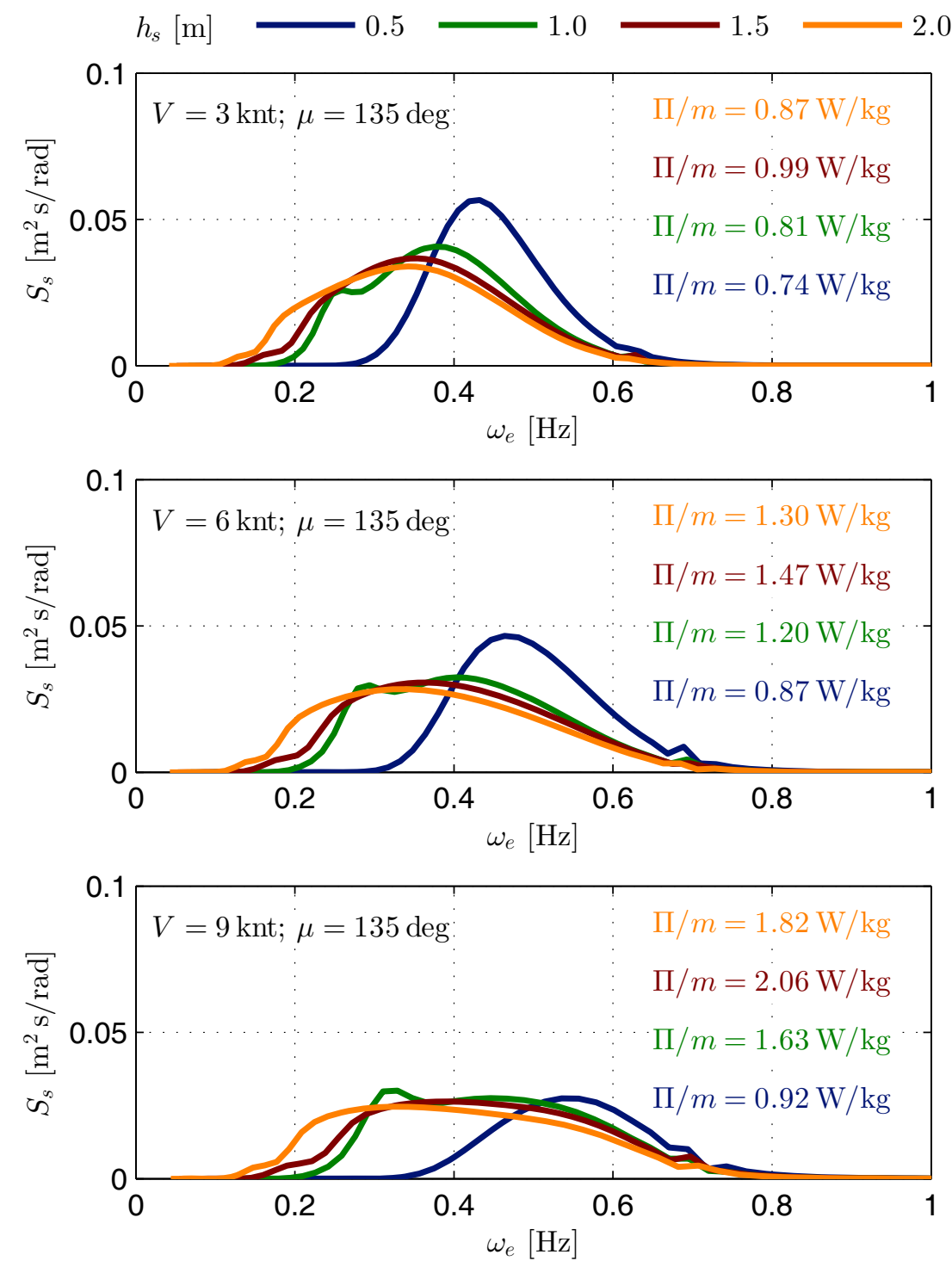

562

Figure 9: Spectral density of the generator's vertical oscillation and average power $(\mu=$ $135 \mathrm{deg})$. 


\begin{tabular}{ccccc}
\hline$h_{s}[\mathrm{~m}]$ & $\alpha$ & $\gamma$ & $f(\alpha, \gamma)$ & $T_{p}[\mathrm{~s}]$ \\
\hline 0.5 & 0.016 & 1.0 & 13.2 & 2.98 \\
1.0 & 0.008 & 2.0 & 14.9 & 4.75 \\
1.5 & 0.010 & 0.5 & 15.5 & 6.06 \\
2.0 & 0.008 & 0.5 & 16.4 & 7.40 \\
\hline
\end{tabular}

Table 1: Parameters used to represent random sea waves at Capo Linaro near Civitavecchia, Italy. 


\begin{tabular}{lrl}
\hline Property & \multicolumn{2}{c}{ Value } \\
\hline Displacement & 6.531 & $\mathrm{t}$ \\
Volume (displaced) & 6.372 & $\mathrm{t}$ \\
Overall length & 11.5 & $\mathrm{~m}$ \\
Draft amidships & 2.475 & $\mathrm{~m}$ \\
Immersed depth & 3.054 & $\mathrm{~m}$ \\
Waterline length & 10.64 & $\mathrm{~m}$ \\
Max beam on waterline & 2.866 & $\mathrm{~m}$ \\
Max section area & 1.213 & $\mathrm{~m}^{2}$ \\
Waterplane area & 21.21 & $\mathrm{~m}^{2}$ \\
Prismatic coefficient (Cp) & 0.494 & \\
Block coefficient (Cb) & 0.068 & \\
\hline
\end{tabular}

Table 2: Hydrostatic properties. 


\begin{tabular}{|c|c|c|c|c|c|c|}
\hline \multirow[b]{3}{*}{$h_{s}[\mathrm{~m}]$} & \multicolumn{6}{|c|}{$y_{0 s}[\mathrm{~m}]$} \\
\hline & \multicolumn{2}{|c|}{$V=3 \mathrm{knt}$} & \multicolumn{2}{|c|}{$V=6 \mathrm{knt}$} & \multicolumn{2}{|c|}{$V=9 \mathrm{knt}$} \\
\hline & $\mu=90^{\circ}$ & $\mu=135^{\circ}$ & $\mu=90^{\circ}$ & $\mu=135^{\circ}$ & $\mu=90^{\circ}$ & $\mu=135^{\circ}$ \\
\hline 0.5 & 0.386 & 0.306 & 0.377 & 0.298 & 0.349 & 0.290 \\
\hline 1.0 & 0.702 & 0.611 & 0.689 & 0.619 & 0.646 & 0.634 \\
\hline 1.5 & 0.975 & 0.874 & 0.959 & 0.884 & 0.913 & 0.906 \\
\hline 2.0 & 1.177 & 1.098 & 1.161 & 1.106 & 1.131 & 1.129 \\
\hline
\end{tabular}

Table 3: Yacht's significant vertical oscillation amplitudes at the generator's location. 


\begin{tabular}{|c|c|c|c|c|c|c|}
\hline \multirow[b]{3}{*}{$h_{s}[\mathrm{~m}]$} & \multicolumn{6}{|c|}{$\Pi_{\mathrm{RMS}} / m[\mathrm{~W} / \mathrm{kg}]$} \\
\hline & \multicolumn{2}{|c|}{$V=3 \mathrm{knt}$} & \multicolumn{2}{|c|}{$V=6 \mathrm{knt}$} & \multicolumn{2}{|c|}{$V=9 \mathrm{knt}$} \\
\hline & $\mu=90^{\circ}$ & $\mu=135^{\circ}$ & $\mu=90^{\circ}$ & $\mu=135^{\circ}$ & $\mu=90^{\circ}$ & $\mu=135^{\circ}$ \\
\hline 0.5 & 0.631 & 0.744 & 0.642 & 0.874 & 0.609 & 0.920 \\
\hline 1.0 & 0.588 & 0.815 & 0.586 & 1.197 & 0.546 & 1.629 \\
\hline 1.5 & 0.694 & 0.988 & 0.692 & 1.474 & 0.646 & 2.065 \\
\hline 2.0 & 0.617 & 0.873 & 0.613 & 1.301 & 0.570 & 1.821 \\
\hline
\end{tabular}

Table 4: Average power generation. 


\begin{tabular}{|c|c|c|c|c|c|c|}
\hline \multirow[b]{3}{*}{$h_{s}[\mathrm{~m}]$} & \multicolumn{6}{|c|}{$s_{0 s}[\mathrm{~m}]$} \\
\hline & \multicolumn{2}{|c|}{$V=3 \mathrm{knt}$} & \multicolumn{2}{|c|}{$V=6 \mathrm{knt}$} & \multicolumn{2}{|c|}{$V=9 \mathrm{knt}$} \\
\hline & $\mu=90^{\circ}$ & $\mu=135^{\circ}$ & $\mu=90^{\circ}$ & $\mu=135^{\circ}$ & $\mu=90^{\circ}$ & $\mu=135^{\circ}$ \\
\hline 0.5 & 0.500 & 0.500 & 0.500 & 0.500 & 0.500 & 0.404 \\
\hline 1.0 & 0.500 & 0.500 & 0.500 & 0.500 & 0.500 & 0.500 \\
\hline 1.5 & 0.500 & 0.500 & 0.500 & 0.500 & 0.500 & 0.500 \\
\hline 2.0 & 0.500 & 0.500 & 0.500 & 0.500 & 0.500 & 0.500 \\
\hline
\end{tabular}

Table 5: Generator significant oscillation amplitudes. 


\begin{tabular}{|c|c|c|c|c|c|c|}
\hline \multirow[b]{3}{*}{$h_{s}[\mathrm{~m}]$} & \multicolumn{6}{|c|}{$c / m[\mathrm{~N} \mathrm{~s} /(\mathrm{m} \mathrm{kg})]$} \\
\hline & \multicolumn{2}{|c|}{$V=3 \mathrm{knt}$} & \multicolumn{2}{|c|}{$V=6 \mathrm{knt}$} & \multicolumn{2}{|c|}{$V=9 \mathrm{knt}$} \\
\hline & $\mu=90^{\circ}$ & $\mu=135^{\circ}$ & $\mu=90^{\circ}$ & $\mu=135^{\circ}$ & $\mu=90^{\circ}$ & $\mu=135^{\circ}$ \\
\hline 0.5 & 1.628 & 1.430 & 1.604 & 1.362 & 1.474 & 1.785 \\
\hline 1.0 & 1.934 & 2.081 & 1.874 & 2.525 & 1.682 & 2.944 \\
\hline 1.5 & 2.493 & 2.707 & 2.418 & 3.296 & 2.182 & 3.879 \\
\hline 2.0 & 2.333 & 2.607 & 2.261 & 3.248 & 2.034 & 3.880 \\
\hline
\end{tabular}

Table 6: Optimum damping coefficients for random wave excitation. 


\begin{tabular}{|c|c|c|c|c|c|c|}
\hline \multirow[b]{3}{*}{$h_{s}[\mathrm{~m}]$} & \multicolumn{6}{|c|}{$\beta$} \\
\hline & \multicolumn{2}{|c|}{$V=3 \mathrm{knt}$} & \multicolumn{2}{|c|}{$V=6 \mathrm{knt}$} & \multicolumn{2}{|c|}{$V=9 \mathrm{knt}$} \\
\hline & $\mu=90^{\circ}$ & $\mu=135^{\circ}$ & $\mu=90^{\circ}$ & $\mu=135^{\circ}$ & $\mu=90^{\circ}$ & $\mu=135^{\circ}$ \\
\hline 0.5 & 0.324 & 0.284 & 0.319 & 0.271 & 0.293 & 0.355 \\
\hline 1.0 & 0.385 & 0.414 & 0.373 & 0.502 & 0.335 & 0.586 \\
\hline 1.5 & 0.496 & 0.539 & 0.481 & 0.656 & 0.434 & 0.772 \\
\hline 2.0 & 0.464 & 0.519 & 0.450 & 0.646 & 0.405 & 0.772 \\
\hline
\end{tabular}

Table 7: Optimum damping ratios for random wave excitation. 